\title{
双 8一羖基喹啉系絡合高聚物的电子順磁共振波譜*
}

\author{
徐元植鲁宪謀
}

（中国科学院化学物理研究所）

金属有机絡合物結构的研究，具有重要的理論 和实际的意义。电子順磁共振是研究金属有机絡合 物結构的有力的工具之一。从近期文献 ${ }^{[1-3]}$ 报导看 来, 这方面工作给处于开拓阶段, 抹且对于某些电 子順磁共振現像和波偝还未能提供足够的数据, 因 而在波墙的解析上还有許多困难。这方面的工作向 有待于大量数据的积糸。

我們測得了, 以双8-美基埕啉衍生物为配位单 体的金属有机絡合高聚物的电子順磁共振波譜, 是 交献上向未拫导过的。其通式为:

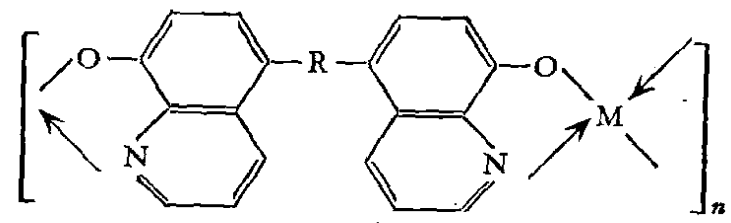

式中 $\mathrm{R}$ 为 $-\mathrm{N}=\mathrm{N}-\mathrm{N}=\mathrm{N}-\mathrm{N}=\mathrm{N}-$ 、 - $\mathrm{CH}_{2}-$ 。为了方便起見, 我們将上述三种不同 $\mathrm{R}$ 的单体, 依次記作 $N 、 P 、 O$ 。式中的 $M$ 为: $B e$, $\mathrm{Mg}, \mathrm{Al},(\mathrm{VO}), \mathrm{Cr}, \mathrm{Mn}, \mathrm{Fe}, \mathrm{Co}, \mathrm{Ni}, \mathrm{Cu}, \mathrm{Zn}, \mathrm{Ag}$, $\mathrm{Cd}, \mathrm{Pb}$ 等不同价态的离子 (見表 1)。

样品的制备和純化在前交 ${ }^{[4]}$ 中已經报导过。

所用波湔仪为国产順磁-2 型, 諧振腔为 $\mathrm{H}_{011}$ 型; 頻率为 9,145 兆周/秒; 灵敏度为 $10^{-9}$ 克分子 $\mathrm{DPPH}$; 分辩率为 0.25 高斯。

样品的 $\mathrm{g}$ 值, 鼠旋浓度和綫宽，都是与标准的 DPPH 的波譜比較得到的。所得数据列于表 1 中。

我們从实驗結果发現， $\mathrm{N}$ 单体中 $\mathrm{Fe}{ }^{++}$的絡合 高聚物是一条筧綫，約 1,000高斯，而在中間大約 $g=2.0$ 处夹着一条很窄的譚綫, 如图 1 所示。这

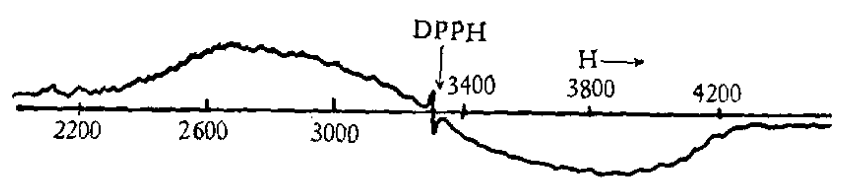

图 $1 \mathrm{Fe}^{++}$的 $\mathrm{N}$ 单体絡合高聚物的电子順磁共振波譜 与 $\mathrm{Cr}_{2} \mathrm{O}_{3}-\mathrm{SiAl}$ 催化剂上看到的波譜[5]很策，但却不 能用它来作相似的解释。(VO $)^{++}$在 N 和 P 两种单 体的絡合高聚物中的波熋綫型是相似的，如图 2 所 示。 $\mathrm{Cu}^{++}$在三种刍体的高聚物中的波鉴也是相似 的, 如图 3 所示。 $\mathrm{Zn}^{++}$在三种解体中的波熋都是一 条窄綫，其 $\mathrm{g}$ 值接沂于 2.0 ，如图 4 所示。

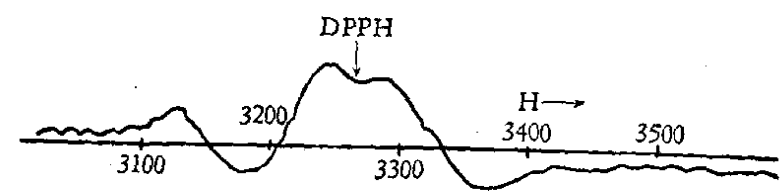

图 $2(\mathrm{VO})^{++}$的 $\mathrm{N}$ 单体絡合高聚物的电子順磁共振波譜

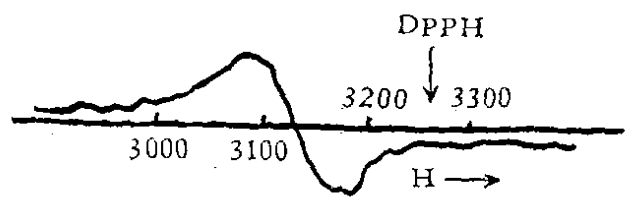

图 $3 \mathrm{Cu}^{++}$的 P 单体絡合高聚物的 电子顧磁共振波謓

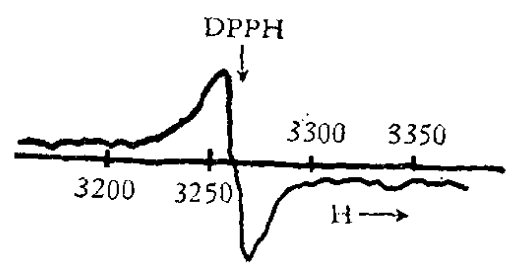

图 $4 \mathrm{Zn}^{++}$的 P 单体絡合高聚物的 电子順磁共振波譜

* 本交曾在全国第一次物盾結狥报告会上报告过。 
表 1 双 8-帮基喹啉系金属絡合高聚物的电子順磁共振波諸

\begin{tabular}{|c|c|c|c|c|c|c|c|c|c|}
\hline \multirow{2}{*}{ 金属 } & \multicolumn{3}{|c|}{$\mathrm{N}$ 单 体 } & \multicolumn{3}{|c|}{$\mathrm{P}$ 单 体 } & \multicolumn{3}{|c|}{ O 单 体 } \\
\hline & $g$-因子 & $\begin{array}{c}\text { 自旋㳖度 } \\
\text { (自旋数/克) }\end{array}$ & $\begin{array}{l}\text { 綫，寬 } \\
\text { (高斯) }\end{array}$ & $g$-因子 & $\begin{array}{c}\text { 自旋浓度 } \\
\text { (自旋数/克) }\end{array}$ & $\begin{array}{l}\text { 綫寬 } \\
\text { (高斯) }\end{array}$ & g-因子 & $\begin{array}{c}\text { 自旋浓度 } \\
\text { (自旋数/克) }\end{array}$ & $\begin{array}{l}\text { 綫 寬 } \\
\text { (高斯) }\end{array}$ \\
\hline $\mathrm{Be}^{++}$ & 2.0 & $6 \times 10^{17}$ & 5 & - & 无訊号 & - & - & 未研究 & - \\
\hline $\mathrm{Mg}^{++}$ & - & 未硥究 & - & 2.0 & $2 \times 10^{17}$ & 5 & - & 未犐究* & - \\
\hline $\mathrm{Al}^{++}+$ & - & 末研究 & 一 & - & 无訊号 & 一 & - & 末研究 & 一 \\
\hline$(\mathrm{VO})^{++* *}$ & - & 一条寬綫 & - & - & 一条寬綫 & - & - & 未㸴究 & - \\
\hline $\mathrm{Cr}^{++}$ & - & 米研究 & 一 & - & 无訊号 & 一 & - & 未研究 & 一 \\
\hline $\mathrm{Mn}^{++}$ & 2.0 & $1 \times 10^{17}$ & 5 & - & 无訊号 & - & - & 未研究 & - \\
\hline $\mathrm{Fe}^{++}$ & - & 一条筧綫 & 一 & - & 无訊号 & - & - & 未研究 & 一 \\
\hline $\mathrm{Co}^{+H}$ & 2.0 & $1 \times 10^{17}$ & 5 & - & 无訊号 & - & - & 未呼究 & - \\
\hline $\mathrm{Ni+H}$ & 2.0 & $1 \times 10^{17}$ & 5 & $一$ & 无訊号 & - & - & 无訊号 & - \\
\hline $\mathrm{Cu}^{++}$ & 2.1 & $3 \times 10^{17}$ & 150 & 2.1 & $1 \times 10^{18}$ & 150 & 2.1 & $1 \times 10^{18}$ & 150 \\
\hline $\mathrm{Ag}^{+}+$ & - & 寺研究 & - & 2.0 & $1 \times 10^{17}$ & 5 & - & 末研究 & - \\
\hline $\mathrm{Zn+}$ & 2.0 & $1 \times 10^{18}$ & 5 & 2.0 & $1 \times 10^{18}$ & 5 & 2.0 & $1 \times 10^{17}$ & 5 \\
\hline $\mathrm{Cd}^{++}$ & 2.0 & $1 \times 10^{18}$ & 5 & 2.0 & $1 \times 10^{18}$ & 5 & - & 未䂰究* & - \\
\hline $\mathrm{Pb}^{++}$ &. & 未研究 & - & - & 无訊号 & - & - & 未研究 & - \\
\hline 沿存金属 & - & 无訊号 & - & - & 无訊号 & 一 & - & 无訊号 & - \\
\hline
\end{tabular}

注 * Бельский ${ }^{[8]}$ 研究过, 自旋浓度为 $2 \times 10^{17}$ 自旋/克。 ** $(\mathrm{VO})^{++}$中是 $\mathrm{V}^{++++}$与 $\mathrm{O}$ 生成共价鍵。

从表中数据看出：三种单 体本身 是没 有 訊号 的，看来訊号应該是由金属所引起的。但从金属离 子的电子組态来看, $\mathrm{Be}^{++} 、 \mathrm{Mg}^{++} 、 \mathrm{Al}^{+++} 、 \mathrm{Zn}^{++}$、 $\mathrm{Cd}^{++}$都是沟有未配对电子的，而在 $\mathrm{N}$ 单 体的絡合 高聚物中，除 $\mathrm{Mg}^{++} 、 \mathrm{Al}^{+++}$未研究过以外，都是有 訊号的。区之, $\mathrm{Cr}^{+++} 、 \mathrm{Mn}^{++} 、 \mathrm{Fe}^{++} 、 \mathrm{Co}^{++} 、 \mathrm{Ni}^{++}$ 都是有未配对电子的，但却在 $\mathrm{P}$ 单体的絡合高聚物 中, 是沟有訊号的。而象 $\mathrm{Cu}^{++} 、 \mathrm{Zn}^{++}$以及 $\mathrm{Cd}^{++}$ $\left(\mathrm{Cd}^{++}\right.$在 $\mathrm{O}$ 单体的数据是引用交献 [3] 的), 在三 种单体中全都有訊号。Бельский ${ }^{[3]}$ 世发現 $\mathrm{Mn}^{++}$、 $\mathrm{Co}^{++} 、 \mathrm{Ni}^{++} 、 \mathrm{Cu}^{++}$在O单体絡合高聚物中是沟有窄 訊号的, 而 $\mathrm{Zn}^{++} 、 \mathrm{Cd}^{++} 、 \mathrm{Hg}^{++}$和 $\mathrm{Mg}^{++}$則都有窄 訊一号。迄今为止, $\mathrm{Zn}^{++} 、 \mathrm{Cd}^{++}$在所有被研究过的单 体中 ${ }^{[2,3]}$ 都发現有訊号，且都还沒有提出过确切的 解析。

我們还研究过双 8-垟基喹啉銅和鎳的电子順磁 共振波譜，

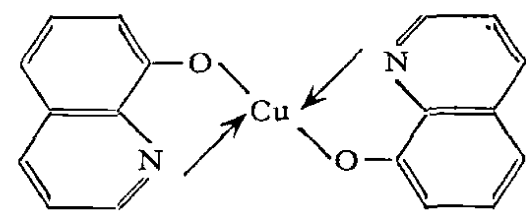

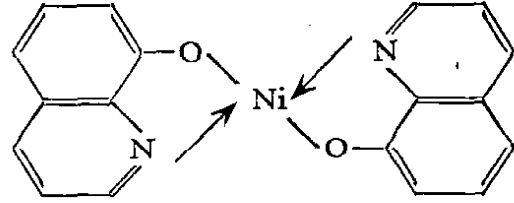

发現前者是有訊号的，而后者則无。

高聚物分子量的大小对訊号强弱产生影响是可 能的 ${ }^{[3]}$ 。但是由于我們对样品的精制純化和波譔的 重复出現，排除了其他杂稹引起訊号的可能性。

至于存在未配对电子而没有訊号的原因是复杂 的, 可能是在配位場的作用下, 次外层的未配对电 子的磁短受到屏蔽, 或是由于高聚物分子本身象乱 銅絲一样，而分子中包含許多个金属原子，其电子 磁矩的取向是凌乱的，且相互制約，或者是由于自 旋晶格的强烈相互作用，而使波謭变得太窅，以致 振幅低到使我們波譜仪的灵钽度难以发現等等，台 有待于进一步探討。

关于 $\mathrm{Be}^{++} 、 \mathrm{Mg}^{++} 、 \mathrm{Zn}^{++} 、 \mathrm{Cd}^{++}$等絡合高聚物 的訉号来源問題，从 $g \simeq 2.0$ 看来是純自旋的未配 对电子所引起的。可能与高聚物的大共 澶体系有 关 ${ }^{6]}$ 。

与电导的数据 ${ }^{[}{ }^{4}$ 比較, 发現有一致的趋势, 即 
有訊号的多牛电阻率較低, 但在 P 单体中却也不尽 然, 如 $\mathrm{Mg}^{++} 、 \mathrm{Zn}^{++} 、 \mathrm{Cd}^{++}$是有訊号的, 电阻率很 大；而 $\mathrm{Mn}^{++} 、 \mathrm{Fe}^{++} 、 \mathrm{Co}^{++} 、 \mathrm{Ni}^{++}$是沟有訊号的, 电 阻却較小，可見电导性能扭不簡单地依賴于順磁共 振的訊号。

金属有机絡合物的电子順磁共振波證出現异常 的現像，表明在金属有机絡合物化学中，有着許多 重要而有趣的課題。工作厼在进行中。

[1] Матвеева Н. Г., Хим. Промышил., № 12, 897
(1962).

[2] Бельский Н. К. и Цикунов В. Н., ДАН СССР., 142, 380 (1962).

[3] Бельский Н. К. и Цикунов В. Н., Высокомолек. соед., 5, 754 (1963).

[4]㲘宪謀、余淑文、徐仁賢, 中国科学院高分子学术 会議会刊, 286 頁, 科学出版社, 1961。

[5] Казанский В. Б. и др., Кинетика и катализ, 2, 454 (1961).

[6] Блюменфельд А. А., Воеводский В. В., Семенов А. Г., Применение электронного парамагнитного резонанса в химии, стр. 216, Изд. Сибир. Отд. АН СССР, Новосибирск, 1962.

\title{
凗乙酸单晶 $\gamma$ 綫輻照电子自旋共振譜的研究
}

\author{
郭德 良
}

关于有机单晶 $r$ 輻炤电子自旋共振的研究近年 来已有不少的报导 ${ }^{[1-8]}$ 。多数的研究仅是二元酸及 氨基酸等化合物。例如 $\mathrm{HOOCCH}_{2}-\mathrm{X}, \mathrm{X}=\mathrm{COOH}$; $\left(\mathrm{CH}_{2}\right)_{n} \mathrm{COOH}, n=1,2,3,4$; $\mathrm{NH}_{2} ; \mathrm{NHCOOH}$; 以及 $\mathrm{NHCO}-\mathrm{NH}_{2}$ 等。經 $r$ 輻照后，打掉了 $\alpha$ 碳上 的貭子，形成了 $\mathrm{HOOC}-\dot{\mathrm{C}} \mathrm{H}-\mathrm{X}$ 自由基。对于未 成对电子与 $\alpha$ 稹子的构形相互作用及 $\beta$ 稹子的超共 䡉作用研究已較完整。但与邻近碱核的相互作用研 究較少。而对 $\mathrm{X}=\mathrm{CN}$ 郎 $\mathrm{NCCH}_{2} \mathrm{COOH}$ 的工作至 今文献上向未見到报导，本文企图通过䕜乙酸单晶 $r$ 輻照电子自旋共振的研究, 探討未成对电子与 $\mathrm{CN}$ 鍵上 $\mathrm{N}$ 的耦合以及基的結构。

㲵乙酸单晶是按下述方法培羕: 将㲵乙酸溶于 水中, 放在干燥器內, 在室温慢慢蒸发, 长成如图 1 所示的晶体, 其大小为 $a=3 \mathrm{~mm} 、 b=2.5 \mathrm{~mm}$ 、 $c=5 \mathrm{~mm}$ 。

单晶在空溫用 $\mathrm{Co}^{60} \mathrm{r}$ 綫进行輻炤, 剂率为 222 伦琴/秒, 輻照时間 5 小时, 剂量为 $3.99 \times 10^{6}$ 帒 琴, 輻照后晶体变为紫色。然后在窒溫用苏联的

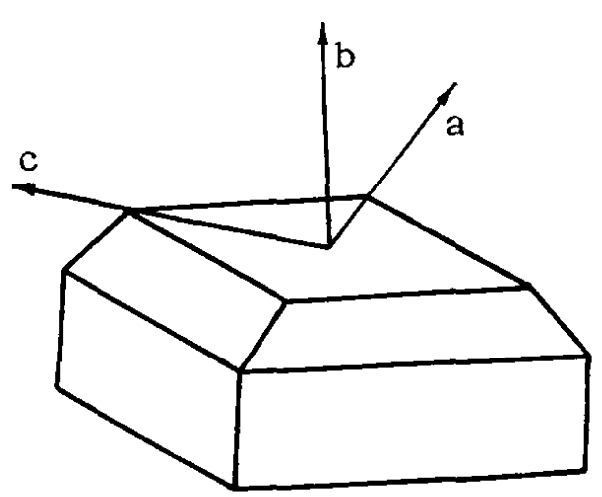

图 1. 诵乙酸单晶外形及所选的 $a b c$ 正交坐标系

ЭПР-2 型順磁共振仪进行測定。以 $\mathrm{Mn}^{++}$（在 $\mathrm{ZnS}$ 中）六条謭綫中的第三与第四两条 綫之閏的寬度 67.6 高斯作为測定磁場的标尺, 及其中心点 $\mathrm{g}$ 値 2.0060 作为測定单晶 $g$ 值的基准。

当磁場垂直于 $a$ 嵮, 处于 $c b$ 平面內, 抹由 $c$ 軸 向 $b$ 軸轉动 $30^{\circ}$ (記为 $a c b 30^{\circ}$ ) 得到如图 2 所示的 波譜。譜綫分成二組，每一組及分裂成相等强度的 三条綫。这表明未成对电子与稹子 $(I=1 / 2)$ 耦合 形成二条綫, 进一步与 $\mathrm{N}^{14}(I=1)$ 耦合又分裂为 